\title{
A Note on Ideals and Regularity in Ternary Semigroups
}

\author{
Anila Peposhi \\ Department of Mathematical Engineering, \\ Polytechnic University of Tirana, Tirana, Albania \\ anila87@outlook.com
}

\begin{abstract}
In this paper we have studied some properties of ideals in ternary semigroups. We have also studied some properties of regular ternary semigroups. We have presented some definitions and prepositions related to them.
\end{abstract}

\section{Keywords}

Ternary Semigroup, Left (Right, Lateral) Ideal, Regular Ternary Semigroup,

\section{INTRODUCTION}

J. Los[6] studied some properties of ternary semigroups and proved that every ternary semigroup can be embedded in a binary semigroup. D.H. Lehmer[5] gave the definition of a ternary semigroup.Banach showed by an example that a ternary semigroup does not necessarily reduce to a binary semigroup. F.M. Sioson[4] studied ideal theory in ternary semigroups and gave the definitions of ideals. The notion of regularity was introduced and studied by J.von Neumann [7] in 1936. F.M.Sioson [4] introduced the notion of regular ternary semigroups.

\section{PRELIMINARIES}

\subsection{Definition}

A ternary semigroup is a non-empty set $S$ together with a ternary operation which has the property of association:

$$
(a b c) d e=a(b c d) e=a b(c d e)
$$

for all $a, b, c$ and $d$ in $S$.

\subsubsection{Example}

The set $\{-i, 0, i\}$ is a ternary semigroup under the multiplication over complex number and is not a binary semigroup under this multiplication.

\subsection{Definition}

A non-empty set $T$ of a ternary semigroup $S$ is called a subsemigroup of $S$ if $a \in T, b \in T$ and $c \in T$ imply $a b c \in T$.

\subsection{Definition}

A ternary semigroup $S$ is called:

(i) left cancellative if $a b x=a b y$ implies $x=y$ for all $a, b, x, y$ in $S$ (ii) right cancellative if $x a b=y a b$ impliesx $=y$ for all $a, b, x, y$ in $S$

(iii) lateral cancellative if $a x b=a y b$ impliesx $=y$ for all $a, b, x, y$ in $S$

(iv)cancellative if $S$ is left, right and lateral cancellative.

\subsection{Definition}

An element $e$ of a ternary semigroup $S$ is called:

(i) left identity of $S$ if eea $=a$ for all $a$ in $S$

(ii) right identity of $S$ if aee $=$ afor all $a$ in $S$

(iii) lateral identity of $S$ if $e a e=a$ for all $a$ in $S$

(iv) two sided identity of $S$ if $e$ is left and right identityof $S$

$(v)$ identity of $S$ if $e$ is left, right and lateral identity of $S$.

\subsection{Definition}

An element $z$ of ternary semigroup $S$ is called a zero element of $S$ if $z a b=z z a=z a z=a z b=a b z=a z z=z$ for all $a, b$ in $S$

\subsubsection{Example}

In the closed interval $I=[0,1]$ we define $x y z=\min (x, y, z)$ for all $x, y, z \in I$.

Let we show that 0 is a zero element and 1 is an identity of $S$. For all $x \in I, 1 x x=\min \{1, x, x\}=x, x 1 x=\min \{x, 1, x\}=x$, $x x 1=\min \{x, x, 1\}=x, 11 x=\min \{1,1, x\}=x, 1 x 1=$ $\min \{1, x, 1\}=x$ dhe $1 x x=\min \{1, x, x\}=x$. Moreover, for all $c, y \in I \quad$ we have $0 x y=\min \{0, x, y\}=0, x 0 y=\min \{x, 0, y\}=0, x y 0=$ $\min \{x, y, 0\}=0, x 00=\min \{x, 0,0\}=0,0 x 0=$ $\min \{0, x, 0\}=0$ and $x 00=\min \{x, 0,0\}=0 \square$

\subsection{Preposition}

Let $X$ be any set and (o)a ternary operation in $X$ such that $x$ o y o $z=z$ for all $x, y$, $z$ in $X$.Then, $X$ is a ternary semigroup under the operation $(o)$.

\subsubsection{Proof}

For all $x, y, z, u, w$ in $X$, ( о о yо z) o uо $w=z$ o иo $w=w$, $x \circ$ ( $y \circ$ o z o u) ow $=$ xо ио $w=w$ and $x$ o yo $(z$ o u o $w)=x$ o y $о w=w \square$ A ternary semigroup $X(o)$ is called a right zero ternary semigroup in $X$.

In a similar way one may prove that: 


\section{A Note on Ideals and Regularity in Ternary Semigroups}

\subsection{Preposition}

Let $X$ be any set and(*) a ternary operation in $X$ such that $x *$ $y * z=x$ for all $x, y, z$ in $X$. Then, $X$ is a ternary semigroup under the operation $(*) \square$

A ternary semigroup $X(*)$ is called a left zero ternary semigroupin $X$.

\subsection{Preposition}

Let $S$ be a left zero ternary semigroup. Then $S$ is right cancellative.

\subsubsection{Proof}

Let $a, b, x, y$ in $S$ such that $x a b=y a b$. Since $S$ is a left zero ternary semigroup we have $x a b=x$ and $y a b=y$. Thus $x=y$. $\square$ In a similar way one may prove that:

\subsection{Preposition}

Let $S$ be a right zero ternary semigroup. Then $S$ is left cancellative.

\subsection{Preposition}

An element $e$ of a ternary semigroup $S$ is called idempotent if eee $=e$

It is clear that a zero element and identity are idempotent elements. The converse is not true in general.

The sets $\{0\},\{1\}$ and $\{e\}$, where $e$ is an idempotent element, are ternary subsemigroups of $S$.

\subsection{Preposition}

Le $e$ be an idempotent element of a left cancellative ternary semigroup $S$. Then $e$ is a left identity of $S$.

\subsubsection{Proof}

For all $a \in S$, eea $=$ eeeeee $a$ and since $S$ is left cancellative we have $a=e e e a$. Also

eee $=e$ imply $a=e e a \square$

In a similar way one may prove that:

\subsection{Preposition}

Le $e$ be an idempotent element of a right cancellative ternary semigroup $S$. Then $e$ is a right identity of $S$. $\square$

\subsection{Preposition}

Let $S_{1}$ and $S_{2}$ be two ternary semigroups. The map $f: S_{1} \rightarrow S_{2}$ is calleda homomorphism if $f(a b c)=f(a) f(b) f(c)$.

\section{IDEALS IN TERNARY SEMIGROUP}

\subsection{Definition}

A non-empty subset $A$ of a ternary semigroup $S$ is called:

(i)left ideal of $S$ if $S S A \subseteq A$

(ii)right ideal of $S$ if $A S S \subseteq A$

(iii)lateral ideal of $S$ if $S A S \subseteq A$

(iv)two sided ideal of $S$ if $A$ is a left and right ideal of $S$

(v)ideal of $S$ if $A$ is a left, right and lateral ideal of $S$.

\subsection{Definition}

An ideal $I$ of a ternary semigroup $S$ with zero0is calledproper if $I \neq\{0\}$ dhe $I \neq S$

\subsection{Definition}

A ternary semigroup $S$ is called:

(i) left simple if $\mathrm{S}$ has no proper left ideals (ii) right simple if S has no proper right ideals

(iii) lateral simple if $S$ has no proper lateral ideals

(iv) simple if $\mathrm{S}$ has no proper ideals.

It is clear that every left [ right, lateral, two sided, ideal] ideal of a ternary semigroup $S$ is a ternary subsemigroup of $S$.

\subsection{Preposition}

Let $S$ be a ternary semigroup. Then, $S S a$ is a left ideal of $S$, for all $a$ in $S$.

\subsubsection{Proof}

For all $x, y, s_{1}, s_{2} \in S$ we have $s_{1} s_{2}(x y a)=\left(s_{1} s_{2} x\right) y a \in S S a \square$ In a similar way one may prove that:

\subsection{Preposition}

Let $S$ be a ternary semigroup. Then, $a S S$ is a right ideal of $S$, for all $a$ in $S$.

\subsection{Preposition}

A ternary semigroup $S$ is left simple if and only if $S S a=S$ for all $\operatorname{ain} S$

\subsubsection{Proof}

For all $a$ in $S, S S a \subseteq S$. Preposition 2.4.implies that $S S a$ is a left ideal of $S$ and since $S$ is left simple we have $S S a=S$.

Conversely, let $L$ be a left ideal of $S$. For alla $\in L$ we have $S S a=$ $S$. This implies that for all $x \in S, x=b c a$ with $b, c \in S$ and since $L$ is a left ideal of $S$ we have $x \in L$. Thus $S \subseteq L$. We have also that $L \subseteq S$. Hence $L=S$

In a similar way one may prove that:

\subsection{Preposition}

A ternary semigroup $S$ is right simple if and only if $a S S=S$ for all $a$ in $S$.

\subsection{Definition}

Let $S$ be a ternary semigroup and $a$ an element of $S$.

$(i)(a)_{l}=a \cup S S a$ is called the principal left ideal of $S$ generated by $a$

(ii) $(a)_{r}=a \cup a S S$ is called the principal right ideal of $S$ generated by $a$

(iii) $(a)_{m}=a \cup S a S \cup S S a S S$ is called the principal lateral ideal of $S$ generated by $a$

$(i v)(a)=a \cup S S a \cup a S S \cup S a S \cup S S a S S$ is called the principal ideal of $S$ generated by $a$.

\subsection{Preposition}

Let $S$ be a ternary semigroup. Then, for all $a \in S,(a)_{r} S S=$ $a S S$ and $S S(a)_{l}=S S a$.

\subsubsection{Proof}

First let we show that $(a)_{r} S S=a S S$. It is clear that $a S S \subseteq(a)_{r} S S$, for all $a \in S$. Now let $x \in(a)_{r} s_{1}, s_{2} \in S$.

If $x=a$ then $x s_{1} s_{2}=a s_{1} s_{2} \in a S S$.

If $\quad x \in a S S, x=a s_{3} s_{4}$ with $s_{3}, s_{4} \in S$. Then, $x s_{1} s_{2}=$ $\left(a s_{3} s_{4}\right) s_{1} s_{2}=a s_{3}\left(s_{4} s_{1} s_{2}\right) \in a S S$. Thus $(a)_{r} S S=a S S$. Now let we show that $S S(a)_{l}=S S a$. It is clear that $S S a \subseteq S S(a)_{l}$. Let $s_{1}, s_{2} \in S$ and $x \in(a)_{l}$. If $x=a, s_{1} s_{2} x=s_{1} s_{2} a \in S S a$. If $x \in S S a, x=s_{3} s_{4}$ awith $s_{3}, s_{4} \in S$. Then $s_{1} s_{2} x=s_{1} s_{2}\left(s_{3} s_{4} a\right)=\left(s_{1} s_{2} s_{3}\right) s_{4} a \in S S a$. HenceSS $(a)_{l} \subseteq S S a$. $\square$ 


\subsection{Preposition}

Let $S$ be a ternary semigroup, $L$ a left ideal of $S$ and $X$ a nonempty subset of $S$. Then, $L X X$ is a left ideal of $S$.

\subsubsection{Proof}

Let $a \in L, x_{1}, x_{2} \in X$ and $s_{1}, s_{2} \in S$. Then, $s_{1} s_{2}\left(a x_{1} x_{2}\right)=$ $\left(s_{1} s_{2} a\right) x_{1} x_{2} \in L X X$ sinces $s_{1} s_{2} a \in L$ due to the fact that $L$ is a left ideal of $S$

In a similar way one may prove that:

\subsection{Preposition}

Let $S$ be a ternary semigroup , $R$ a right ideal of $S$ and $X$ a nonempty subset of $S$. Then, $X X R$ is a right ideal of $S$.

\subsection{Preposition}

Let $S$ be a ternary semigroup, $L$ a left ideal of $S, R$ a right ideal of $S$ and $M$ a lateral ideal of $S$. Then, $L M R$ is a two sided ideal of $S$. Moreover $R M L \subseteq R \cap M \cap L$.

\subsubsection{Proof}

Let $a \in L, b \in M, c \in R$ and $s_{1}, s_{2} \in S$. Then, $s_{1} s_{2}(a b c)=$ $\left(s_{1} s_{2} a\right) b c \in L M R$ sinces $s_{1} s_{2} a \in L$ due to the fact that $L$ is a left ideal of $S$. Thus $L M R$ is a left ideal of $S$. We have also that $(a b c) s_{1} s_{2}=a b\left(c s_{1} s_{2}\right) \in L M R$ since $c s_{1} s_{2} \in R$ due to the fact that $R$ is a right ideal of $S$. Thus $L M R$ is a right ideal of $S$. Now let we show that $R M L \subseteq R \cap M \cap L$. Let $a \in R, b \in$ $M$ and $c \in L$. Then, $a b c \in R$ since $R$ is a right ideal of $S$. We have also that $a b c \in M$ sinceMis a lateral ideal of $S$. On the other side $a b c \in L$ since $L$ is a left ideal of $S$. This implies that $a b c \in$ $R \cap M \cap L$

\subsection{Preposition}

Let $A$ be a two sided ideal of a ternary semigroup $S$ and $B$ a two sided ideal of $A$ such that $B^{3}=B$. Then, $B$ is a two sided ideal of $S$.

\subsubsection{Proof}

Since $B^{3}=B$ we have that $S S B=S S B B B \subseteq S S A A B \subseteq A A B \subseteq B$. This implies that $B$ is a left ideal of $S$. We have also that $B S S=$ $B B B S S \subseteq B A A S S \subseteq B A A \subseteq B$. This implies that $B$ is a right ideal of $S$.

\subsection{Definition}

Let $S$ be a ternary semigroup. An ideal $I$ of $S$ is called:

(i) strongly irreducible if $I_{1} \cap I_{2} \subseteq$ Iimplies $I_{1} \subseteq I$ or $I_{2} \subseteq I$

(ii) weakly irreducible if $I_{1} \cap I_{2}=I$ implies $I_{1}=I$ or $I_{2}=I$, for any two ideals $I_{1}$ and $I_{2}$ of $S \square$

\subsection{Definition}

Let $S$ be aternary semigroup. An ideal $P$ of $S$ is called:

(i) prime ideal of $S$ if $I_{1} I_{2} I_{3} \subseteq P$ implies $I_{1} \subseteq P$ or $I_{2} \subseteq P$ or $I_{3} \subseteq P$ for any three ideals $I_{1}, I_{2}, I_{3}$ of $S$

(ii) completely prime ideal of $S$ if $x y z \in P$ implies $x \in P$ or $y \in P$ or $z \in P$ for any three elements $x, y, z$ of $S$

\subsection{Definition}

Let $S$ be a ternary semigroup. An ideal $T$ of $S$ is called:

(i) semiprimeif $I I \subseteq T$ Timplies $I \subseteq T$ for any ideal $I$ of $S$

(ii )completelysemiprimeif $x x x \in T \operatorname{implies} x \in T$ for any element $x$ of $S$

\section{REGULARITY IN SEMIGROUPS \\ TERNARY}

4.1 Definition

A ternary semigroup $S$ is called regular if for all $a \in S$ exists $x, y \in S$ such that $a=$ axaya.

It is clear that in a ternary semigroup $S$ we have $S S S=S$.

\subsection{Definition}

An ideal $I$ of a ternary semigroup $S$ is called regular if $I U$ $R M L=R \cap M \cap L$ for any right ideal $R \supseteq I$, lateral ideal $M \supseteq I$, left ideal $L \supseteq I$.

\subsection{Preposition}

Every regular and strongly irreducible ideal is prime.

\subsubsection{Proof}

Let $S$ be a ternary semigroup and $I$ a regular and strongly irreducible ideal of $S$. Let $I_{1} I_{2} I_{3} \subseteq I$. Then , $I_{1} I_{2} I_{3} \cup I=I$. Also, since $I$ is a regular ideal we have $I_{1} I_{2} I_{3} \cup I=I_{1} \cap I_{2} \cap I_{3} \subseteq I$ and since $I$ is strongly irreducible we have $I_{1} \subseteq I$ or $I_{2} \subseteq I$ or $I_{3} \subseteq I$. When cel is prime.

\subsection{Preposition}

Every regular ideal is semiprime.

4.4.1 Proof

Let $\mathrm{S}$ be a ternary semigroup and I a regular ideal of S. Let $J J J \subseteq I$. Then, JJJ $\cup I=I$. Since $I$ is a regular ideal we have $\mathrm{JJJ} \cup \mathrm{I}=\mathrm{J} \cap \mathrm{J} \cap \mathrm{J}=\mathrm{J} \subseteq \mathrm{I}$. Hence lis semiprime.

\subsection{Lemma}

Let $\Phi: S \rightarrow T$ be a one-to-one homomorphism of a ternary semigroup $S$ to a ternary semigroup $T$. Then, $\operatorname{im} \Phi$ is regular. If $f$ is an idempotent element of $\operatorname{im} \Phi$, then exist an idempotent element $e$ of $S$ such that $\Phi(e)=f$.

\subsubsection{Proof}

For all $z \in \operatorname{im} \Phi, z=\Phi(a)$ with $a \in S$. Since $S$ is regular, exists $x, y \in S \quad$ such that $a=$ axaya. Then, $\Phi(a)=\Phi(a) \Phi(x) \Phi(a) \Phi(y) \Phi(a) \quad$ since $\Phi$ is homomorphism. Thus $z=z u z v z w i t h u=\Phi(x)$ and $v=\Phi(y)$. This implies that $\operatorname{im} \Phi$ is regular. Now let $f$ be an idempotent element of $\operatorname{im} \Phi$. Then, $f f f=f$ and exist $e \in \operatorname{Ssuch}$ that $\Phi(e)=$ $f$. Thus $f=\Phi(e) \Phi(e) \Phi(e)=\Phi(e e e)$ and since $\Phi$ is one-to-one we haveeee $=e$.

\subsection{Preposition}

Let $S$ a regular ternary semigroup, $R$ a right ideal of $S, L$ a left ideal of $S$ and $M$ a lateral ideal of $S$. Then, $R M L=R \cap M \cap L$.

4.6.1 Proof

Preposition 2.12.implies $R M L \subseteq R \cap M \cap L$. Let $a \in R \cap M \cap L$. Since $S$ is regular exists $x, y \in S$ such that $a=$ axaya. Since $a \in M$ and $M$ is a lateral ideal of $S$ we have $x a y \in M$. We have also that $a \in R$ and $a \in L$. Hence $a \in R M L$. 


\section{CONCLUSION}

Many concepts in binary semigroups extend similarly to ternary semigroups. In this section we presented some properties of ideals in ternary semigroups and regular ternary semigroups. All of these help us to find out the structure of a ternary semigroup.

\section{REFERENCES}

[1] A.H. Clifford, G.B. Preston, The Algebraic Theory of Semigroups Vol 1, American Mathematical Society (1961)

[2] John M. Howie, Fundamentals of Semigroup Theory, Clarendon Press Oxford(1995)

[3] Otto Steinfeld, Quasi-Ideals in Rings and Semigroups, Akademia Kiado, Budapest (1978)

[4] F.M. Sioson, Ideal Theory in Ternary Semigroups, Math.Jpn. 10, 63-84 (1965)

[5] D.H. Lehmer, A Ternary Analogue of Abelian Groups, Am.J.Math. 54, 329-338 (1932)

[6]J.Los, On the Extending of Models I.Fundam.Math.42, 38-54 (1955)

[7] J.von Neumann, On Regular Rings, Proc. Nat. Acad. Sci. USA 22, 707-713 (1936) 